Diseases

Editorial Manager(tm) for European Journal of Clinical Microbiology \& Infectious

$$
\text { Manuscript Draft }
$$

Manuscript Number: EJCMID-D-10-00039R1

Title: Impact of antibiotic therapy on systemic cytokine expression in pneumococcal pneumonia

Article Type: Article

Keywords: pneumococcal pneumonia, cytokines, antibiotic therapy,IL-6, outcome.

Corresponding Author: Dr. Carolina Garcia-Vidal,

Corresponding Author's Institution:

First Author: Susana Padrones

Order of Authors: Susana Padrones; Carolina Garcia-Vidal; Silvia Fernández-Serrano; Ana Fernández; Cristina Masuet; Jordi Carratalà; Mercè Coromines; Carmen Ardanuy; Francesc Gudiol; Frederic Manresa; Jordi Dorca

Abstract: Purpose: The aim of this study was to compare the evolution of systemic cytokine levels over time in patients with pneumococcal pneumonia treated either with ß-lactam monotherapy or with combination therapy (ß-lactam plus fluoroquinolone).

Methods: Prospective observational study of hospitalized non-immunocompromised adults with PP. Concentrations of IL-6, IL-8, IL-10 and TNF- $\alpha$ were determined on days $0,1,2,3,5$, and 7. Patients on $ß$-lactam monotherapy were compared with those receiving combination therapy.

Results: Fifty-two patients were enrolled in the study. Concentrations of IL-6, IL-8, and IL-10 decreased rapidly in the first days after admission, in accordance with the mean time to defervescence. High levels of IL- 6 were found in patients with the worst outcomes, measured by the need for intensive care unit admission and mortality. No major differences in demographic or clinical characteristics or severity of disease were found between patients treated with ß-lactam monotherapy or combination therapy. IL-6 levels fell more rapidly in patients with combination therapy in the first 48 hours $(\mathrm{p}=0.016)$.

Conclusions: Our data suggest that systemic expression of IL-6 production in patients with PP is correlated with prognosis. Initial combination antibiotic therapy produces a faster decrease in this cytokine in the first 48 hours.

Response to Reviewers: Professor Nele Jung

Editor

European Journal of Clinical Microbiology \& Infectious Diseases

Barcelona, April 19th, 2010

REF: EJCMID-D-10-00039

"Impact of antibiotic therapy on systemic cytokine expression in pneumococcal pneumonia"

Dear Professor Jung: 
We submitted you the revised manuscript entitled "Impact of antibiotic therapy on systemic cytokine expression in pneumococcal pneumonia". We have taken into consideration all the reviewer's suggestions. Please find below a list of responses point by point to the reviewers' comments.

Sincerely, Carolina Garcia-Vidal

Infectious Diseases Service. Hospital Universitari de Bellvitge Feixa llarga s/n. 08907 L'Hospitalet, Barcelona, Spain

E-mail: caroglv75@hotmail.com

Fax: 34932607637

Comments to the Author:

Reviewer \#1: Prospective, non randomized study on the evolution and the impact of antibiotic therapy on systemic cytokine expression in pneumococcal pneumonia.

A total of 52 patients enrolled. Data on the evolution of the systemic cytokine levels obtained every 24 hours during the first 5 days and on day 7. In 39 selected patients, the impact of B-lactam alone vs. Blactam plus fluoroquinolone on the systemic cytokine levels was assessed.

Results showed that high levels of IL- 6 at inclusion predicted the worst outcomes, including admission to ICU and mortality. In the group of patients treated with the combination, IL-6 levels fell more rapidly in the first 48 hours.

\section{COMMENTS}

The study confirms previous reports that have shown a correlation of IL-6 levels at entry and prognosis. The results of the comparative study on the impact of antibiotic therapy showed that combination therapy was associated with a faster decrease in IL- 6 in the first 24 and 48 hours of treatment. The Introduction, Methods and Results are appropriate and pertinent information is provided.

In the Discussion, the authors elaborate considerably on the potential advantages of combination vs. monotherapy in CAP and pneumococcal pneumonia. This is unnecessary. First, this issue is not settled and no randomized trials have been performed to answer this question and, second, as the authors themselves acknowledge, they did not intend to determine whether combination therapy had a better impact on survival than monotherapy. In addition, most studies have used the combination of B-lactam plus macrolide, not B-lactam plus fluroquinolone.

R: According to the reviewer's comment, we have deleted the sentences discussing the potential advantages of combination versus monotherapy. We have added information regarding the point that most studies have used the combination of B-lactam plus macrolide, not B-lactam plus fluroquinolone (page 20, lines 21 and 22).

Also, they state that the role of fluoroquinolone monotherapy as empirical treatment for severe CAP has not been established. This is not correct. The IDSA/ATS guidelines recommend precisely fluoroquinolne monotherapy as one of the options. Besides, they should not refer to CAP, they should be addressing the issue of pneumococcal pneumonia.

R: To avoid confudion we have deleted the sentence. 


\author{
ORIGINAL ARTICLE
}

\title{
Impact of antibiotic therapy on systemic cytokine expression in pneumococcal pneumonia
}

\author{
Susana Padrones ${ }^{1}$, Carolina Garcia-Vidal ${ }^{2,6 *}$, Silvia Fernández-Serrano ${ }^{1}$, Ana \\ Fernández ${ }^{1}$, Cristina Masuet ${ }^{3}$, Jordi Carratalà ${ }^{2,6}$, Mercè Coromines ${ }^{4}$, Carmen \\ Ardanuy $^{5,7}$, Francesc Gudiol ${ }^{2,6}$, Frederic Manresa ${ }^{1}$, Jordi Dorca ${ }^{1,7}$ \\ ${ }^{1}$ Respiratory Medicine, ${ }^{2}$ Infectious Disease, ${ }^{3}$ Preventive Medicine, ${ }^{4}$ Immunology, and \\ ${ }^{5}$ Microbiology Departments of Hospital Universitari de Bellvitge, Institut d'Investigació \\ Biomèdica de Bellvitge (IDIBELL), University of Barcelona, L'Hospitalet de Llobregat, \\ Barcelona, Spain, ${ }^{6}$ REIPI (Spanish Network for the Research in Infectious Diseases) and \\ ${ }^{7}$ CIBER de Enfermedades Respiratorias ISCIII, Madrid, Spain
}

Corresponding author: Carolina Garcia-Vidal, MD, Infectious Disease Service, Hospital Universitari de Bellvitge, Feixa Llarga s/n 08907 L'Hospitalet, Barcelona, Spain (carolgv75@hotmail.com); Telephone: 34-932607625; Fax: 34-932607637

Running title: Impact of antibiotic therapy on cytokine expression 


\section{ABSTRACT}

Purpose: The aim of this study was to compare the evolution of systemic cytokine levels over time in patients with pneumococal pneumonia treated either with $\beta$-lactam monotherapy or with combination therapy ( $\beta$-lactam plus fluoroquinolone).

Methods: Prospective observational study of hospitalized non-immunocompromised adults with PP. Concentrations of IL-6, IL-8, IL-10 and TNF- $\alpha$ were determined on days $0,1,2,3,5$, and 7 . Patients on $\beta$-lactam monotherapy were compared with those receiving combination therapy.

Results: Fifty-two patients were enrolled in the study. Concentrations of IL-6, IL-8, and IL-10 decreased rapidly in the first days after admission, in accordance with the mean time to defervescence. High levels of IL-6 were found in patients with the worst outcomes, measured by the need for intensive care unit admission and mortality. No major differences in demographic or clinical characteristics or severity of disease were found between patients treated with $\beta$-lactam monotherapy or combination therapy. IL-6 levels fell more rapidly in patients with combination therapy in the first 48 hours $(p=0.016)$.

Conclusions: Our data suggest that systemic expression of IL-6 production in patients with PP is correlated with prognosis. Initial combination antibiotic therapy produces a faster decrease in this cytokine in the first 48 hours.

Keywords: pneumococcal pneumonia, cytokines, antibiotic therapy,IL-6, outcome. 


\section{INTRODUCTION}

Streptococcus pneumoniae remains a major cause of disease worldwide [1]. Among pneumonia pathogens, it is the most common cause of hospitalization in adults and the most frequent cause of death $[2,3]$. Despite improvements in etiologic diagnosis, effective antibiotic therapy and advances in supportive care, the morbidity and mortality rates associated with pneumococcal pneumonia (PP) remain high. Case-fatality rates for bacteremic pneumococcal pneumonia range between 7 and 35\% [4].

A recent study [5] of the factors associated with early death in patients with communityacquired pneumonia (CAP) reinforces the classical concept that some deaths are closely related to inadequate host response [6]. Excessive cytokine response in patients with severe CAP has been linked with deleterious effects and poor prognosis [7-14]. However, most studies included populations that were heterogeneous in terms of patients, etiologies, and treatment. Significantly, specific studies of the role of cytokine response for predicting poor outcomes in patients with PP are scarce. In recent years, the modulation of the inflammatory response has emerged as a promising concept for improving the outcomes of CAP. Although it has been suggested that different antibiotic classes may have different effects on the systemic expression of cytokine production [1517], information addressing this issue in PP is lacking.

We carried out a prospective study in order to (i) analyze the relationship between systemic expression of cytokine production and outcomes in patients with PP and (ii) compare the evolution of systemic cytokine levels over time in patients treated either with $\beta$-lactam monotherapy or with combination therapy ( $\beta$-lactam plus fluoroquinolone). 


\section{MATERIAL AND METHODS}

Study subjects and study design. The study was carried out in a 900-bed university hospital for adults in Barcelona, Spain. The hospital serves an area of $1,100,000$ inhabitants and admits approximately 24,000 patients per year. All nonimmunocompromised patients with PP who were admitted to the hospital from January 2005 through December 2005 were prospectively recruited and followed up. Patients with neutropenia, HIV infection or transplantation were not included. Concentrations of circulating cytokines were determined for all patients. To assess the effects of treatment on the systemic expression of cytokine production, patients were divided into two groups: those initially treated only with $\beta$-lactams ( $\beta$-lactam group), and those initially treated with combination therapy including $\beta$-lactams plus fluoroquinolone (combination therapy group). This prospective longitudinal observational study was approved by the Ethical Committee of our Institution.

Clinical evaluation and follow-up. At the initial visit, and before starting empirical antibiotic therapy, patients underwent a complete clinical history, physical examination and laboratory testing. Microbiological studies included two sets of blood cultures and sputum Gram stain and culture when available. Urinary antigen detection for $S$. pneumoniae was performed if indicated by the attending physician. Antimicrobial susceptibility was tested by the microdilution method, following the Clinical Laboratory Standard Institute methods and criteria $[18,19]$.

Empiric antibiotic therapy was administered according to the hospital's guidelines, which recommend the administration of a B-lactam agent (ceftriaxone or amoxicillinclavulanate) with or without a fluoroquinolone (levofloxacin). Combination therapy was recommended for patients with clinical suspicion of Legionella or an atypical pathogen, 
or in case of severe CAP in the absence of a demonstrative sputum Gram stain. Levofloxacin monotherapy was allowed for selected cases (i.e., those patients with allergy to ß-lactam agents and no prior quinolone use).

Patients were seen daily during their hospital stay by one or more of the investigators who provided medical advice when requested and recorded demographic characteristics, underlying disease, clinical features, vaccination status, causative agents, therapy, and outcomes in a computer-assisted protocol.

Definitions. PP was diagnosed in patients with signs and symptoms of an acute-onset lower respiratory tract infection, a new infiltrate on chest radiograph, and one or more cultures positive for $S$. pneumoniae obtained from blood, normally sterile fluids, or sputum and/or a positive test for detection of urinary antigen. Only good quality samples of sputum ( $<10$ squamous epithelial cells and $>25$ leucocytes per field) were accepted for processing. S. pneumoniae was identified using standard microbiology procedures. S. pneumoniae antigen in urine was detected by using a rapid immunochromatographic assay (Now ${ }^{\mathrm{TM}}$, Binax, Portland, ME, USA).

Antimicrobial susceptibility was tested by microdilution. S. pneumoniae strains were serotyped. Molecular characterization was performed by pulsed field gel electrophoresis after restriction with $S m a(I)$ and selected strains were analysed by MLST, as previously reported [1].

The diagnosis of septic shock was based on a systolic blood pressure of less than 90 $\mathrm{mmHg}$ and peripheral hypoperfusion with clinical suspicion of uncontrolled infection. Early death was defined as death due to any cause $\leq 48$ hours of hospitalization. Overall mortality was defined as that due to any cause within 30 days of hospitalization. The severity of illness at presentation was quantified using the validated PORT prediction rule for 30-day mortality and medical complications in CAP, as described elsewhere [20]. 
Collection of blood samples and laboratory processing. For all patients, serial venous blood samples were collected at inclusion, immediately prior to the initiation of antibiotic therapy, and on days $1,2,3,5$, and 7 . The blood obtained was placed in tubes containing EDTA, immediately centrifugated, and stored at $-80^{\circ} \mathrm{C}$. The assays were performed by one of the authors (M.C.), who was blinded to the clinical details of individual patients. The circulating levels of cytokines IL-6, IL-8, IL-10 and TNF- $\alpha$ were measured.

IL-6, IL-8, IL-10 and TNF- $\alpha$, concentrations were measured using commercially available kits (GENZYME, Cambridge, Mass). The procedure consisted of a solid-phase chemiluminescent immunometric assay. The standards defined in the operator's manual were applied. The limits of detection were $3 \mathrm{pg} / \mathrm{ml}$ for IL-6, $1 \mathrm{pg} / \mathrm{ml}$ for IL-8, $4 \mathrm{pg} / \mathrm{ml} \mathrm{IL}-10$ and $0.5 \mathrm{pg} / \mathrm{ml}$ for TNF- $\alpha$.

Statistical analysis. To analyze the relationship between systemic expression of cytokine production and the severity of PP we compared serial serum cytokine measurements in patients who had severity markers (bacteremia, ICU admission and mortality) with those who did not. A comparison of serial cytokine measurements was made with the Kruskal-Wallis one-way analysis of variance nonparametric test.

To assess the effects of treatment on the systemic expression of cytokine production, we compared the combination therapy and $\beta$-lactam groups. Patients who initially received other antibiotic treatments were excluded. To detect significant differences between groups we used the chi-square test with continuity correction for categorical variables. Normally distributed data were compared by using unpaired $t$ tests and the MannWhitney U-test was used for analysis of variables with non-normal distribution. Studies evaluating serum concentrations of cytokines over time (the fall-down pattern) were performed using the general linear model for repeated-measures tests, considering both 
within-subject and between-subject factors (differences attributable to antimicrobial therapy). The contrasts selected were "difference" and "polynomic" for within subject factors. The analysis was adjusted for potential confounding variables (use of corticosteroids or ICU admission). The data analyses were performed with SPSS software version 13.0. In all analyses, we considered $P$ values less than 0.05 to be statistically significant.

\section{RESULTS}

Characteristics of patients and evolution of cytokines over time. Fifty-two hospitalized patients with PP were included. Their demographic characteristics and main clinical features are shown in Table 1. The diagnosis of PP was established with the use of one or more of the following methods: blood culture (21 cases), sputum Gram stain and culture (20 cases), urinary antigen test (17 cases), and transthoracic needle aspiration (2 cases). All S. pneumoniae were susceptible to ciprofloxacin (MIC range 0.5 $2 \mu \mathrm{g} / \mathrm{ml}$ ) and to levofloxacin (MIC range $0.5-1 \mu \mathrm{g} / \mathrm{ml}$ ). Using current non-meningeal breakpoints for beta-lactams, all strains were penicillin (MIC range $\leq 0.03-2 \mu \mathrm{g} / \mathrm{ml}$ ), amoxicillin (MIC range $\leq 0.03-2 \mu \mathrm{g} / \mathrm{ml}$ ) and cefotaxime (MIC range $\leq 0.03-1 \mu \mathrm{g} / \mathrm{ml}$ ) susceptible. The most frequent serotypes were 3,1 and 5 , which accounted for $51.4 \%$ of strains. These serotypes were related to ST260 and ST180 for serotype 3, ST306 for serotype 1.

Concentrations of all the cytokines studied were detected in peripheral venous blood samples in all patients, although with a wide range of values. Figure 1 shows the evolution of cytokines over time. At admission, IL-6 and IL-8 showed the highest values. When the variations in concentrations were analyzed over time, all cytokines except 
TNF- $\alpha$, showed a statistically significant trend towards a rapid decrease after 24 to $48 \mathrm{~h}$. TNF- $\alpha$ remained basically unmodified throughout the study period.

Clinical outcomes and their relationship with systemic cytokines. The main outcomes of patients are summarized in table 2. Mean time to defervescence was 2.19 days (SD 1.19). After the initial evaluation in the emergency department, 37 (71.2\%) patients were admitted to a conventional hospital ward, whereas the other $15(28.8 \%)$ were transferred to an intensive care unit (ICU). The median length of ICU stay was 7 days (range 2-72 days). The early and overall case-fatality rates were $1.9 \%$ and $15.4 \%$ respectively. The evolution of systemic cytokines concentration over time in relation to bacteremia was determined, as well as ICU admission and mortality. Table 3 shows serum levels of cytokine on days 0,1 , and 2 in relation to these outcomes. In summary, no significant differences in cytokine levels were found in patients with or without bacteremia. IL-6 was significantly higher in patients requiring ICU admission and in patients who died. High levels of IL-8, especially on day 1, were also documented in patients with ICU admission and in those who died.

On day 0 , the third quartile for IL-6 initial concentrations identified $83.3 \%$ non-survivors and only $16.2 \%$ survivors. Thus, at admission, an IL-6 level $>5206 \mathrm{pg} / \mathrm{ml}$ predicted mortality with a sensitivity of $100 \%$, a specificity of $79.3 \%$, a positive predictive value of $80.6 \%$, and a negative predictive value of $100 \%$. On day 1 , levels of IL-6 $>4097 \mathrm{pg} / \mathrm{ml}$ predicted mortality with a sensitivity of $100 \%$, a specificity of $100 \%$, a positive predictive value of $80 \%$, and a negative predictive value of $94.3 \%$.

Effects of antibiotic treatment on cytokine production. To assess the effects of treatment on systemic cytokine production, we compared 19 patients in the $\beta$-lactam group (ceftriaxone in 15 cases and amoxicillin-clavulanate in four) with 20 patients in the combination therapy group (ceftriaxone plus levofloxacin in all cases). Thirteen patients 
(five initially treated with combination therapy [ $\beta$-lactam plus macrolide], four initially treated with linezolid and four initially treated with a single fluoroquinolone treatment were excluded from the analysis. There were no differences in the characteristics of the patients who were included and those who were excluded. Demographic characteristics, the main clinical features and outcomes of patients by treatment group are shown in Table 4. Interestingly, no important differences in demographic characteristics, vaccination status, time from pneumonia onset to inclusion, previous use of steroids, statins, non-steroidal anti-inflammatory drugs, severity of infection, bacteremia and outcomes were found between groups. Chronic heart and cerebrovascular diseases were more frequent in patients in the $\beta$-lactam group. Conversely, the presence of multilobar infiltrates was more frequent in the combination therapy group.

As shown in figure 2, the IL-6 decrease was more rapid in the combination therapy group, particularly in the first days $(p=0.016)$. TNF- $\alpha$ levels were lower in the combination therapy group, but the differences did not reach statistical significance. No differences in the evolution over time of IL-8 were detected. Conversely, levels of anti-inflammatory cytokines $(\mathrm{IL}-10)$ remained higher $(\mathrm{p}<0.001)$ in the combination therapy group. All these differences remained significant after adjustment for the use of corticosteroids and ICU admission.

\section{DISCUSSION}

Previous studies of CAP have noted that most cytokines can be detected in systemic circulation and show a significant pattern of decline in the first hours of treatment. Indeed, all cytokines studied in the present report were detected in venous blood samples in patients with PP at hospital admission. Thus, most patients developed a systemic extension of the initially compartmentalized immune response in the lung. 
Interestingly, all these cytokines, except for TNF- $\alpha$, declined in the first 48 hours. These decreases correlate clinically with the time to clinical defervescence.

Previous studies have reported that an excess of proinflammatory cytokines is associated with poor prognosis of CAP [7-14]. Our results showed a similar relationship between high levels of cytokines and poor outcomes in the specific population with PP. We demonstrated that levels of IL-6 on admission, as well as levels of II-6 and IL-8 in the first $48 \mathrm{~h}$, were the best markers for predicting poor outcomes, in agreement with previous studies analyzing any etiology of CAP. A recent study ${ }^{11}$ found that the addition of biological markers such as C-reactive protein to severity scoring systems (PSI, CURB65 and CRB-65) improves the 30-day mortality prediction. Further studies are currently needed to establish the potential role of IL-6 and IL-8 in supplementing prognosis scoring systems in order to achieve a more accurate identification of patients with a greater probability of death.

The most notable finding of this study was the difference in the cytokine profile between patients treated with $\beta$-lactam monotherapy and those treated with combination therapy. We found that combination therapy of a $\beta$-lactam plus fluoroquinolone produced a faster decreased in IL-6 in the initial 48 hours of treatment in patients with PP. Taking into account the relationship between IL-6 and poor prognosis for PP, the modulation of the expression of this cytokine may be a key point for improving patient outcomes. Whether combination therapy can improve outcomes in patients with CAP is a controversial issue [21-26]. Moreover, most studies have used the combination of $\beta$-lactam plus macrolide, not $\beta$-lactam plus fluoroquinolone.

A possible explanation for the differences observed in the pattern of systemic cytokine production over time is that $\beta$-lactam cell wall activity causes the release of cell wall components which act as potent inflammatory inducers [27-30]. One hypothesis is that 
combination therapy offers more rapid microbial killing due to the presence of quinolones and hence shortens the exposure of the host to microbial products. Additionally, fluoroquinolones have an intrinsic immunomodulation effect that inhibits the production of certain pro-inflammatory cytokines [15-18,31]. The possible beneficial effects of fluoroquinolones on the systemic expression of cytokine response when combined with $\beta$-lactam therapy in patients with PP have not been previously explored, but our results suggest that their potential anti-inflammatory and immunomodulatory effects persist when combined with $\beta$-lactam. A previous study [8] explored the effects of fluoroquinolone monotherapy in modulating the cytokine response in patients with PP, finding that it achieved a faster decrease in serum TNF- $\alpha$ production at 120 hours postadmission than $\beta$-lactam monotherapy.

Our study has limitations that should be acknowledged. First, the study was observational and included a relatively small number of patients. Second, only four patients were treated with fluoroquinolone monotherapy, precluding comparisons in the pattern of systemic expression of cytokine production. Finally, it should be emphasized that our aim was not to establish whether combination therapy of $\beta$-lactam plus fluoroquinolone has a clear impact on survival in the first hours after admission for PP. Therefore, our results should be interpreted with caution.

We found that IL-6, IL-8, IL-10 were detected in venous blood samples in all patients with PP at hospital admission with a rapid decrease in the first $48 \mathrm{~h}$, correlating with clinical defervescence. High levels of IL-6 were found in patients with the worst outcomes. Initial $\beta$-lactam and fluoroquinolone combination antibiotic therapy produced a faster decrease of this cytokine in the first 48 hours. 


\section{Funding}

This study was supported by research grants of Fundación Pi i Sunyer; FUCAP (Fundació Catalana de Pneumologia); REIPI RD06/0008 from the Ministerio de Sanidad y Consumo, Instituto de Salud Carlos III, Spanish Network for the Research in Infectious Diseases; by FIS (070864); and by Institut d'Investigació Biomèdica de Bellvitge (Dr. Garcia-Vidal).

\section{Transparency declaration}

None to declare 


\section{References}

[1] Ardanuy C, Tubau F, Pallarés R, et al. (2009) Epidemiology of invasive pneumococcal disease among adult patients in Barcelona before and after pediatric 7valent pneumococcal conjugate vaccine introduction 1997-2007. Clin Infect Dis 48:5764.

[2] Barlett JG, Mundy L.M. (1995) Community-acquired pneumonia. N Engl J Med 333:1618-24.

[3] Rosón B, Carratalà J, Dorca J, et al. (2001) Etiology, reasons for hospitalization, risk classes and outcomes of patients with community-acquired pneumonia hospitalized on the basis of conventional admission criteria. Clin Infect Dis 33:158-65.

[4] Feikin DR, Schuchat A, Kolczak M. (2000) Mortality from invasive pneumococcal pneumonia in the era of antibiotic resistance, 1995-1997. Am J Public Health 90:223-9.

[5] Garcia-Vidal C, Fernández-Sabé N, Carratalà J, et al. (2008) Early mortality in patients with community-acquired pneumonia: causes, and risk factors. Eur Respir $\mathrm{J}$ 32:733-9.

[6] Austrian R, Gold J. (1964) Pneumococcal bacteremia with especial reference to bacteremic pneumococcal pneumonia. Ann Intern Med 60:759-70.

[7] Antunes G., Evans SA, Lordan JL, et al. (2002) Systemic cytokine levels in community-acquired pneumonia and their association with disease severity. Eur Respir $\mathrm{J}$ 20:990-5.

[8] Calbo E, Alsina M, Rodríguez-Carballeira M, et al. (2008) Systemic expression of cytokine production in patients with severe pneumococcal pneumonia: effects of treatment with a $\beta$-lactam versus a fluoroquinolone. Antimicrob Agents Chemother 52:2359-402. 
[9] Fernández-Serrano S, Dorca J, Coromines M, et al. (2003) Molecular inflammatory responses measured in blood of patients with severe community-acquired pneumonia. Clin Diag Lab Immunol 10:813-20.

[10] Kellum JA, Kong L, Fink MP, et al. (2007) Understanding the inflammatory cytokine response in pneumonia and sepsis: results of the Genetic and Inflammatory markers of sepsis (GenIMS) study. Arch Intern Med 167:1655-63.

[11] Menendez R, Martinez R, Reyes S, et al. (2009) Biomarkers improve mortality prediction by prognostic scales in community-acquired pneumonia. Thorax 64:587-91.

[12] Monton C, Torres A, El-Ebiary M, et al. (1999) Cytokine expression in severe pneumonia: a bronchoalveolar lavage study. Crit Care Med 27:1745-53.

[13] Örtqvist A, Hedlund J, Wretlind B, et al. (1995) Diagnostic and prognostic value of Interleukin-6 and C-reactive protein in community-acquired pneumonia. Scand J Infect Dis 27:457-62.

[14] Xu F, Droemann D, Rupp J, et al. (2008) Modulation of the inflammatory response to Streptococcus pneumoniae in a model of acute lung tissue infection. Am J Respir Cell Mol Biol 39:522-9.

[15] Demartini G, Esposti D, Marthyn P, et al. (2004) Effect of multiple doses of clarithromycin and amoxicillin on IL-6, IFNgamma and IL-10 plasma levels in patients with community-acquired pneumonia. J Chemother 16:82-5.

[16] Choi J., Song M, Kim G, et al. (2003) Effect of moxifloxacin on production of proinflammatory cytokines from human peripheral blood mononuclear cells. Antimicrob Agents Chemother 47:3704-7.

[17] Dalhoff A, Shalitl I. (2003) Immunomodulatory effect of quinolones. Lancet Infect Dis 3:359-71. 
[18] Clinical Laboratory Standard Institute (CLSI). Performance standards for antimicrobial susceptibility testing: 19th informational supplement. CLSI document M100S18. Wayne, PA: CLSI, 2009.

[19] Clinical and Laboratory Standard Institute (CLSI). Methods for dilution antimicrobial susceptibility test for bacteria that growth aerobically; approved standard: 7th edition. CLSI documentM7-A6. Wayne, PA: CLSI, 2006.

[20] Fine MJ, Auble TE, Yealy DM, et al. (1997) A prediction rule to identify low-risk patients with community-acquired pneumonia. N Engl J Med 336:243-50.

[21] Waterer GW, Somes GW, Wunderink RG. (2001) Monotherapy may be suboptimal for severe bacteremic pneumococcal pneumonia. Arch Intern Med 161:1837-42.

[22] Martinez JA, Horcajada JP, Almela M, et al. (2003) Addition of a macrolide to a betalactam-based empirical antibiotic regimen is associated with lower in-hospital mortality for patients with bacteremic pneumococcal pneumonia. Clin Infect Dis 36:389-95.

[23] Baddour LM, Yu VL, Klugman KP, et al. (2004) Combination therapy lowers mortality among severely ill patients with pneumococcal pneumonia. Am J Respir Crit Care Med 170:440-4.

[24] Rodriguez A., Mendia A, Sirvent JM, et al. (2007) Combination antibiotic therapy improves survival in patients with community-acquired pneumonia and shock. Crit Care Med 35:1493-8.

[25] Harbarth S., Garbino J, Pugin J, et al. (2005) Lack of effect of combination antibiotic therapy on mortality in patients with pneumococcal sepsis. Eur J Clin Microbiol Infect Dis 24:688-90.

[26] Aspa J, Rajas O, Rodriguez de Castro F, et al. (2006) Impact of initial antibiotic choice on mortality from pneumococcal pneumonia. Eur Resp J 27:1010-9. 
[27] Heumann D, Barras C, Severin A, et al. (1994) Gram-positive cell wall stimulates synthesis of tumor necrosis factor alpha and interleukin 6 by human monocytes. Infect Immun 62:2715-21.

[28] Tomasz A, Saukkonen D. (1989) The nature of cell wall derived inflammatory components of pneumococci. Pediatr Infect Dis J 8:902-3.

[29] Tuomanen E, Rich R, Zak O. (1987) Induction of pulmonary inflammation by components of the pneumococcal cell surface. Am Rev Respir Dis 1987;135:869-74.

[30] Tuomanen E, Vanholder R, De Paepe P, et al. (1996) Immunomodulating effects of antibiotics: literature review. Infection 24:275-91.

[31] Blaine T, Pollice P, Rosier R, et al. (1997) Modulation of the production of cytokines in titanium-stimulated human peripheral blood monocytes by pharmacological agents: the role of camp-mediated signaling mechanism. J Bone Joint Surg 79:1519-28. 


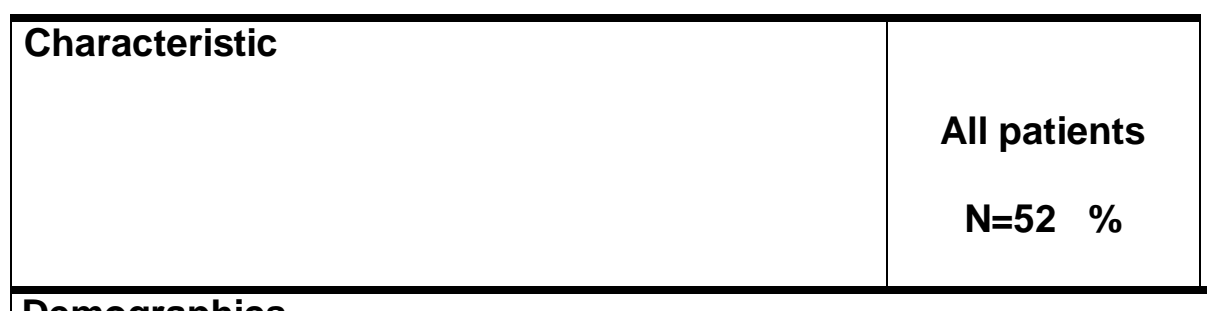

\section{Demographics}

\begin{tabular}{|c|c|c|}
\hline Age, mean years (SD) & \multicolumn{2}{|c|}{$58.8(19.3)$} \\
\hline Male, sex & 34 & 65.4 \\
\hline Current smoker & 21 & 40.4 \\
\hline Heavy drinking & 4 & 7.7 \\
\hline \multicolumn{3}{|l|}{ Vaccination status } \\
\hline Influenza vaccine (season) & 11 & 21.2 \\
\hline Pneumococcal vaccination $^{\mathrm{e}}$ & 4 & 7.7 \\
\hline \multicolumn{3}{|l|}{ Underlying disease } \\
\hline COPD $^{\dagger}$ & 14 & 26.9 \\
\hline Chronic heart disease & 10 & 19.2 \\
\hline Diabetes mellitus & 9 & 17.3 \\
\hline Cerebrovascular disease & 4 & 7.7 \\
\hline Chronic liver disease & 1 & 1.9 \\
\hline Chronic renal disease & 0 & 0 \\
\hline Time from pneumonia onset to inclusion & \multicolumn{2}{|c|}{$2(0-15)$} \\
\hline Previous use of statins & 6 & 11.5 \\
\hline Concomitant use of steroids & 2 & 3.8 \\
\hline Concomitant use of $\mathrm{NSAl}^{*}$ drugs & 5 & 9.6 \\
\hline High risk PSI (IV-V) & 31 & 59.6 \\
\hline Clinical features & & \\
\hline
\end{tabular}




\begin{tabular}{|l|c|c|}
\hline Altered mental status on admission & 11 & 21.1 \\
\hline Renal failure (Cr > 150 mmol/L) & 15 & 28.8 \\
\hline Urea median mmol/dl (range) & \multicolumn{2}{|c|}{$9.5(2-30)$} \\
\hline Heart rate mean (SD) & $104.5(19.20)$ \\
\hline Respiratory rate mean (SD) & \multicolumn{2}{|c|}{$33.5(9.6)$} \\
\hline Temperature median (range) & $38.5(36-40)$ \\
\hline Leucocytes mean (SD) & $14431(7406)$ \\
\hline PO2/fiO2 ${ }^{\dagger}$ mean (SD) & $243(43.8)$ \\
\hline PO2/fiO2 ${ }^{\dagger}<300$ & 43 & 82.7 \\
\hline Multilobar infiltrates & 24 & 46.2 \\
\hline Shock at admission & 7 & 13.5 \\
\hline Pleural effusion & 12 & 23.1 \\
\hline
\end{tabular}

*nonsteroidal anti-inflammatory drug 
Figure 1. Sequential cytokine levels in patients with severe pneumococcal pneumonia. All cytokines except TNF- $\alpha$, showed a statistically significant trend towards a rapid decrease after 24

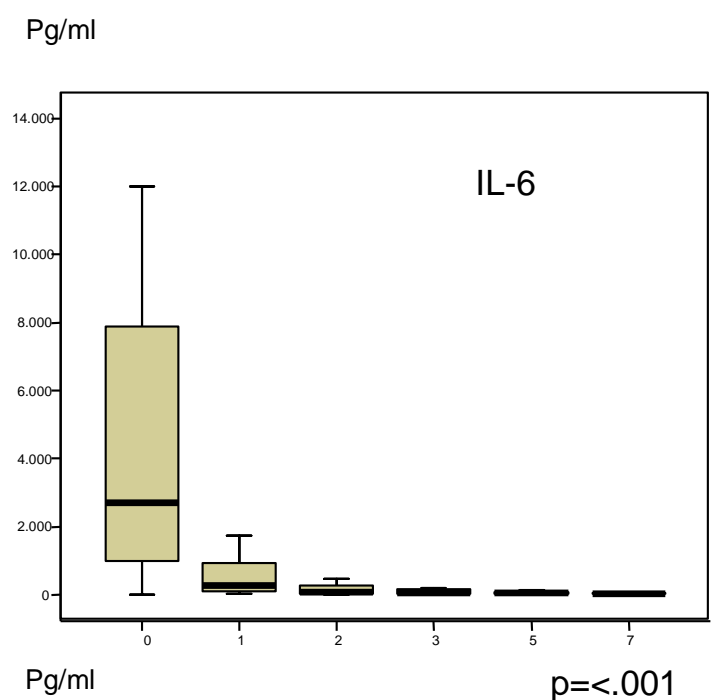

$$
\mathrm{Pg} / \mathrm{ml}
$$
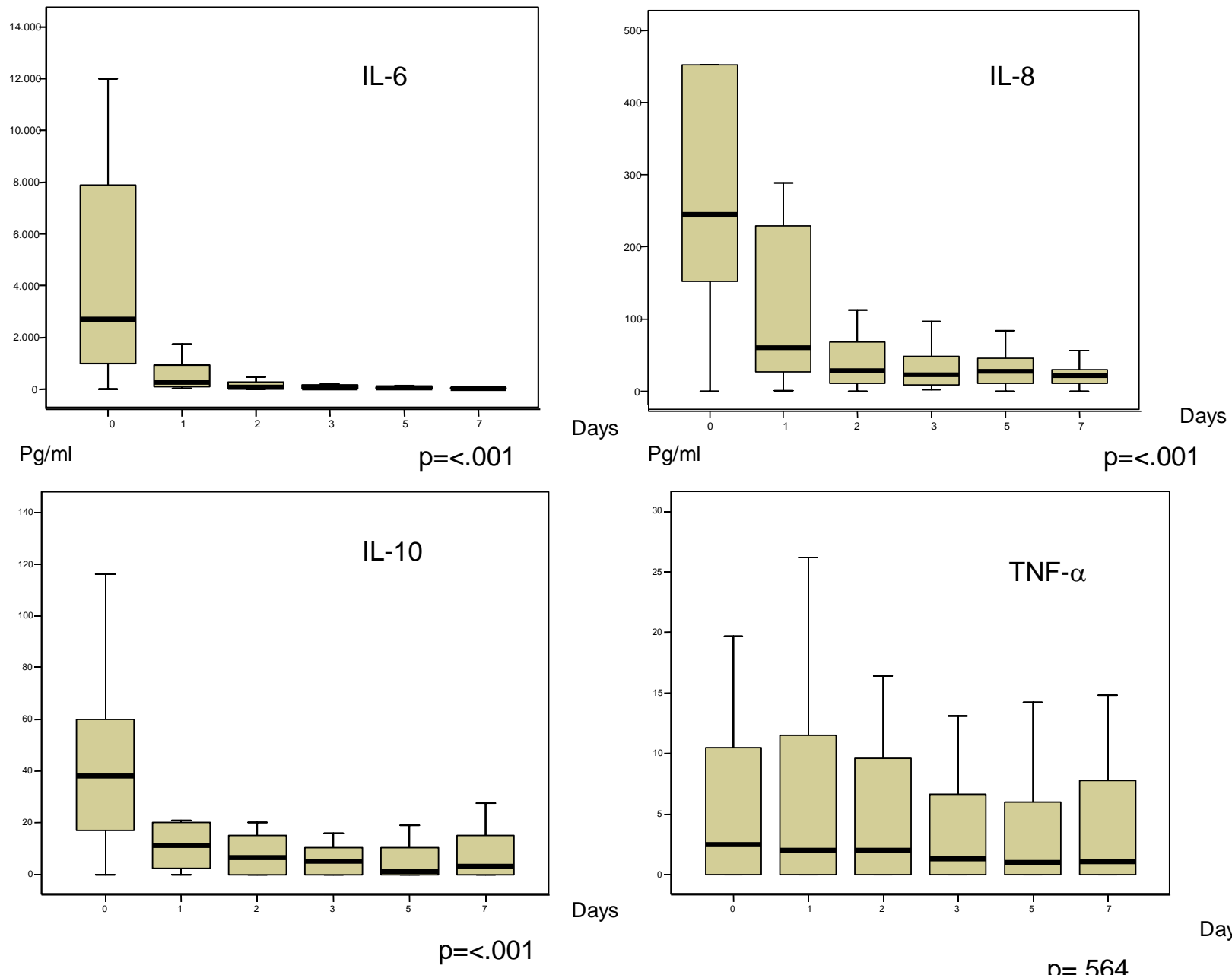

$\mathrm{p}=.564$ 
Table 2. Outcomes of pneumococcal pneumonia of patients hospitalized for community-acquired pneumonia.

\begin{tabular}{|l|c|c|}
\hline Outcomes & \multicolumn{2}{|c|}{ All } \\
& $n=52$ & 36.8 \\
\hline Bacteremia & 21 & 29 \\
\hline ICU admission & 15 & 17.3 \\
\hline Length of ICU admission median (range) & \multicolumn{2}{|c|}{$5.5(2-72)$} \\
\hline Need for mechanical ventilation & 9 & 5.7 \\
\hline Non-invasive mechanical ventilation & 3 & 11.5 \\
\hline Invasive mechanical ventilation & 6 & 1.9 \\
\hline Mechanical ventilation-free days median (range) & \multicolumn{2}{|c|}{$6.5(2-72)$} \\
\hline Early mortality & 1 & 15.4 \\
\hline Overall mortality & 8 & \\
\hline
\end{tabular}


Table 3. Results of cytokines on days 0 and 1 in the groups with or without bacteremia, with or without ICU admission and deaths or survivors.

\begin{tabular}{|c|c|c|c|c|c|c|c|c|c|}
\hline \multirow[b]{2}{*}{ Cytokine } & \multicolumn{3}{|c|}{ Bacteremia } & \multicolumn{3}{|c|}{ ICU admission } & \multicolumn{3}{|c|}{ Mortality } \\
\hline & $\begin{array}{l}\text { No }(n=31) \\
\text { Median }\left(P_{25}-75\right)\end{array}$ & $\begin{array}{l}\text { Yes }(\mathrm{n}=21) \\
\text { Median }\left(\mathrm{P}_{25^{-75}}\right)\end{array}$ & $p$ & $\begin{array}{l}\text { No }(\mathrm{n}=37) \\
\text { Median }\left(\mathrm{P}_{25^{-75}}\right)\end{array}$ & $\begin{array}{l}\text { Yes }(n=15) \\
\text { Median }\left(P_{25^{-75}}\right)\end{array}$ & $p$ & $\begin{array}{c}\text { Survivors }(\mathrm{n}=46) \\
\text { Median }\left(\mathrm{P}_{25-75}\right)\end{array}$ & $\begin{array}{c}\text { Deaths }(\mathrm{n}=8) \\
\text { Median }\left(\mathrm{P}_{25^{-75}}\right)\end{array}$ & $p$ \\
\hline \multicolumn{10}{|l|}{ Day 0} \\
\hline IL-6 & $\begin{array}{c}2945 \\
(919-4550)\end{array}$ & $\begin{array}{c}2700 \\
(778-10335)\end{array}$ & NS & $\begin{array}{c}1890 \\
(850-4488)\end{array}$ & $\begin{array}{c}10335 \\
(2700-21160)\end{array}$ & .028 & $\begin{array}{c}2190 \\
(835-4492)\end{array}$ & $\begin{array}{c}8878 \\
(6236-18453)\end{array}$ & .020 \\
\hline IL-8 & $\begin{array}{c}181 \\
(67-307) \\
\end{array}$ & $\begin{array}{c}152 \\
(43-975) \\
\end{array}$ & NS & $\begin{array}{c}165 \\
(54-256) \\
\end{array}$ & $\begin{array}{c}452 \\
(43-1675) \\
\end{array}$ & NS & $\begin{array}{c}180 \\
(51-392) \\
\end{array}$ & $\begin{array}{c}148 \\
(48-5953)\end{array}$ & NS \\
\hline IL-10 & $\begin{array}{c}23 \\
(13-84) \\
\end{array}$ & $\begin{array}{c}32 \\
(9-60) \\
\end{array}$ & NS & $\begin{array}{c}20 \\
(2-63) \\
\end{array}$ & $\begin{array}{c}47 \\
(29-207) \\
\end{array}$ & NS & $\begin{array}{c}26 \\
(13-64)\end{array}$ & $\begin{array}{c}91 \\
(12-259) \\
\end{array}$ & NS \\
\hline TNF- $\alpha$ & $\begin{array}{c}3 \\
(0.2-9.5)\end{array}$ & $\begin{array}{c}13 \\
(0-19)\end{array}$ & NS & $\begin{array}{c}3 \\
(0-10)\end{array}$ & $\begin{array}{c}12 \\
(2-19)\end{array}$ & NS & $\begin{array}{c}3 \\
(0-11)\end{array}$ & $\begin{array}{c}11 \\
(4-17)\end{array}$ & NS \\
\hline \multicolumn{10}{|l|}{ Day 1} \\
\hline IL-6 & $\begin{array}{c}276 \\
(99-1146)\end{array}$ & $\begin{array}{c}753 \\
(234-2375)\end{array}$ & NS & $\begin{array}{c}237 \\
(99-848)\end{array}$ & $\begin{array}{c}2700 \\
(1405-16029)\end{array}$ & $<.001$ & $\begin{array}{c}276 \\
(107-850)\end{array}$ & $\begin{array}{c}8992 \\
(2347-21518)\end{array}$ & $<.001$ \\
\hline IL-8 & $\begin{array}{c}62 \\
(19-229)\end{array}$ & $\begin{array}{c}39 \\
(16-164)\end{array}$ & NS & $\begin{array}{c}36 \\
(15-76)\end{array}$ & $\begin{array}{c}288 \\
(176-837)\end{array}$ & $<.001$ & $\begin{array}{c}41 \\
(19-165)\end{array}$ & $\begin{array}{c}217 \\
(55-1689)\end{array}$ & .050 \\
\hline IL-10 & $\begin{array}{c}12 \\
(3-19)\end{array}$ & $\begin{array}{c}10 \\
(14-73)\end{array}$ & NS & $\begin{array}{c}11 \\
(4-19)\end{array}$ & $\begin{array}{c}51 \\
(5-203)\end{array}$ & NS & $\begin{array}{c}11 \\
(3-19)\end{array}$ & $\begin{array}{c}66 \\
(3-232)\end{array}$ & NS \\
\hline TNF- $\alpha$ & $\begin{array}{c}3 \\
(0-5)\end{array}$ & $\begin{array}{c}11 \\
(1.2-15)\end{array}$ & NS & $\begin{array}{c}3 \\
(0-11)\end{array}$ & $\begin{array}{c}5 \\
(0.5-11)\end{array}$ & NS & $\begin{array}{c}3 \\
(0-12)\end{array}$ & $\begin{array}{c}6 \\
(0-19)\end{array}$ & NS \\
\hline \multicolumn{10}{|l|}{ Day 2} \\
\hline IL-6 & $\begin{array}{c}94 \\
(24-217)\end{array}$ & $\begin{array}{c}87 \\
(66-458)\end{array}$ & NS & $\begin{array}{c}66 \\
(24-112)\end{array}$ & $\begin{array}{c}308 \\
(118-1626)\end{array}$ & $<.001$ & $\begin{array}{c}85 \\
(30-228)\end{array}$ & $\begin{array}{c}2522 \\
(1091-5246)\end{array}$ & .001 \\
\hline IL-8 & $\begin{array}{c}24 \\
(9-76)\end{array}$ & $\begin{array}{c}22 \\
(3-39)\end{array}$ & NS & $\begin{array}{c}20 \\
(9-54)\end{array}$ & $\begin{array}{c}54 \\
(2-92)\end{array}$ & NS & $\begin{array}{c}20 \\
(6-59)\end{array}$ & $\begin{array}{c}66 \\
(30-508)\end{array}$ & NS \\
\hline IL-10 & $\begin{array}{c}7 \\
(0-13)\end{array}$ & $\begin{array}{c}7 \\
(0-25)\end{array}$ & NS & $\begin{array}{c}7 \\
(0-13)\end{array}$ & $\begin{array}{c}9 \\
(3-65)\end{array}$ & NS & $\begin{array}{c}7 \\
(0-12)\end{array}$ & $\begin{array}{c}38 \\
(0-104)\end{array}$ & NS \\
\hline TNF- $\alpha$ & $\begin{array}{c}2 \\
(0-9)\end{array}$ & $\begin{array}{c}10 \\
(2-16.4)\end{array}$ & NS & $\begin{array}{c}2 \\
(0-13)\end{array}$ & $\begin{array}{c}6 \\
(2-12)\end{array}$ & NS & $\begin{array}{c}3 \\
(0-12)\end{array}$ & $\begin{array}{c}2 \\
(0-15)\end{array}$ & NS \\
\hline
\end{tabular}


Table 4. Demographic characteristics, main clinical features and outcomes of patients by treatment group.

\begin{tabular}{|c|c|c|c|c|c|}
\hline Characteristic & \multicolumn{2}{|c|}{$\begin{array}{c}\beta \text {-lactams } \\
\text { group } \\
N=19\end{array}$} & \multicolumn{2}{|c|}{$\begin{array}{l}\text { Combination } \\
\text { therapy group } \\
\qquad N=20\end{array}$} & $\mathbf{p}$ \\
\hline \multicolumn{6}{|l|}{ Demographics } \\
\hline Age, median years (range) & \multicolumn{2}{|c|}{$63.3(18.0)$} & \multicolumn{2}{|c|}{$56.5(17.0)$} & .232 \\
\hline Male, sex & 14 & 73.7 & 12 & 60 & .501 \\
\hline Current smoker & 6 & 31.6 & 9 & 47.4 & .508 \\
\hline Heavy drinking & 3 & 15.8 & 1 & 5 & 287 \\
\hline \multicolumn{6}{|l|}{ Vaccination status } \\
\hline Influenza vaccine (season) & 4 & 21.0 & 5 & 25.0 & .317 \\
\hline Pneumococcal vaccination $^{\mathrm{e}}$ & 1 & 5.2 & 3 & 15.0 & .699 \\
\hline \multicolumn{6}{|l|}{ Underlying disease } \\
\hline $\mathrm{COPD}^{\dagger}$ & 7 & 36.8 & 4 & 20.0 & .160 \\
\hline Chronic heart disease & 9 & 47.4 & 0 & 0 & $<.001$ \\
\hline Diabetes mellitus & 4 & 21.1 & 3 & 15.0 & .451 \\
\hline Cerebrovascular disease & 3 & 15.8 & 0 & 0 & .036 \\
\hline Chronic liver disease & 1 & 5.3 & 0 & 0 & .241 \\
\hline Chronic renal disease & 0 & 0 & 0 & 0 & 1 \\
\hline Time from pneumonia onset to inclusion & \multicolumn{2}{|c|}{$2.5(0-15)$} & \multicolumn{2}{|c|}{$3(1-15)$} & .975 \\
\hline Previous use of statins & 3 & 15.8 & 2 & 10.0 & .661 \\
\hline Concomitant use of steroids & 2 & 10.5 & 0 & 0 & .230 \\
\hline Concomitant use of $\mathrm{NSAl}^{*}$ drugs & 3 & 15.8 & 0 & 0 & .106 \\
\hline High risk PSI (IV-V) & 13 & 68.4 & 14 & 70 & 1 \\
\hline
\end{tabular}




\section{Clinical features}

\begin{tabular}{|c|c|c|c|c|c|}
\hline Altered mental status on admission & 4 & 21.0 & 3 & 15.0 & .451 \\
\hline Renal failure $(\mathrm{Cr}>150 \mathrm{mmol} / \mathrm{L})$ & 8 & 42.1 & 5 & 25.0 & .320 \\
\hline Urea median mmol/dl (range) & \multicolumn{2}{|c|}{$11(2-30)$} & \multicolumn{2}{|c|}{$11(5-25)$} & .538 \\
\hline Respiratory rate mean (SD) & \multicolumn{2}{|c|}{$31.9(8.3)$} & \multicolumn{2}{|c|}{$36.9(9.1)$} & .123 \\
\hline Temperature median (range) & \multicolumn{2}{|c|}{$38.5(37.7-38.2)$} & \multicolumn{2}{|c|}{$38.5(36-40)$} & .813 \\
\hline $\mathrm{PO}^{2 / f i O}{ }^{\dagger}$ mean (SD) & \multicolumn{2}{|c|}{$238.0(43.0)$} & \multicolumn{2}{|c|}{$232.6(42.6)$} & .760 \\
\hline $\mathrm{PO}_{2} / \mathrm{fiO}^{\dagger}<300$ & 16 & 84.2 & 18 & 90 & .146 \\
\hline Multilobar infiltrates & 2 & 10.5 & 18 & 90 & $<0.001$ \\
\hline Shock at admission & 3 & 15.8 & 3 & 15.0 & .408 \\
\hline Pleural effusion & 3 & 15.8 & 5 & 25.0 & .727 \\
\hline Bacteremia & 8 & 42.1 & 6 & 30.0 & .325 \\
\hline \multicolumn{6}{|l|}{ Outcomes } \\
\hline ICU admission & 6 & 31.6 & 6 & 30.0 & 1 \\
\hline Need for mechanical ventilation & 3 & 15.8 & 4 & 20.0 & .732 \\
\hline Early mortality & 1 & 5.3 & 0 & 0 & .299 \\
\hline Overall mortality & 3 & 15.8 & 5 & 25.0 & .694 \\
\hline
\end{tabular}

*nonsteroidal anti-inflammatory drugs 
Figure 2. Evolution of IL-6 and II-10 systemic concentrations by treatment group. IL-6 decreased faster in the combination therapy group $(p=0.016)$. Conversely, levels of anti-inflammatory cytokines were higher in this group $(p<0.001)$. This analysis has been adjusted for potential confounder variables (i.e. the use of corticosteroids or ICU admission)

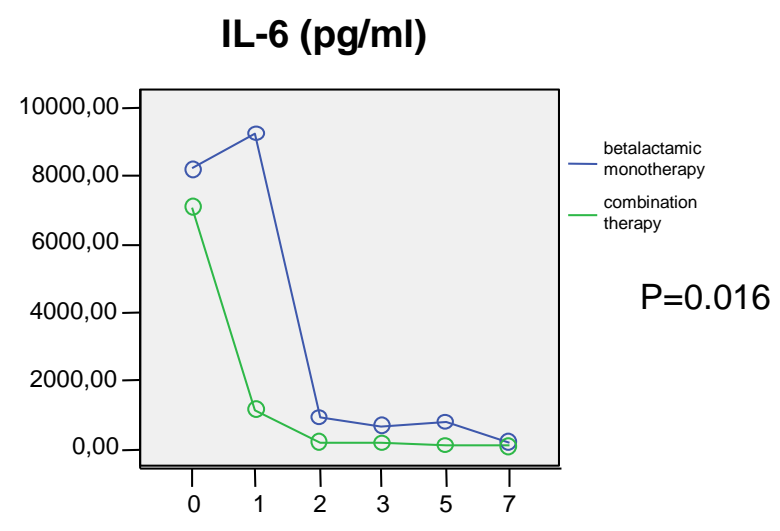

Days

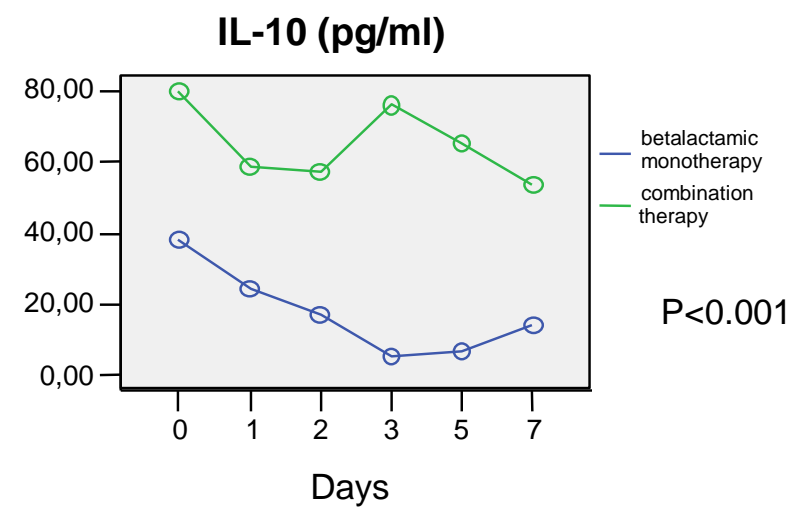

